\title{
Error-detection-coding-aided iterative hard decision interference cancellation for MI MO systems with HARQ
}

\author{
Sangjoon Park ${ }^{1 *}$ \\ ${ }^{1}$ Department of Information and Communication Engineering, Wonkwang University \\ Iksan, Jeonbuk, 54538 - Korea, \\ [e-mail: sjpark24@wku.ac.kr] \\ *Corresponding author: Sangjoon Park
}

Received March 20, 2017; revised July 15, 2017; accepted October 29, 2017;

published March 31, 2018

\begin{abstract}
In this paper, an error-detection-coding-aided iterative hard decision interference cancellation (EDC-IHIC) scheme for multiple-input multiple-output systems employing hybrid automatic repeat request (HARQ) for multi-packet transmission is developed and investigated. In the EDC-IHIC scheme, only packets identified as error-free by the EDC are submitted to the interference cancellation (IC) stage for cancellation from the received signals. Therefore, the possibility of error propagation, including inter-transmission error propagation, can be eliminated using EDC-IHIC. Because EDC must be implemented in systems that employ HARQ to determine packet retransmission, error propagation can be prevented without the need for additional redundancy. The results of simulations conducted herein verify that the EDC-IHIC scheme outperforms conventional hard decision IC schemes in terms of the packet error rate in various environments.
\end{abstract}

Keywords: MIMO systems, hybrid ARQ, hard decision interference cancellation, error detection coding

This paper was supported by Wonkwang university in 2017 . 


\section{Introduction}

Interference cancellation (IC) schemes [1]-[9] are a reception technique for multiple-input multiple-output (MIMO) systems; these schemes can achieve a high level of performance with reduced computational complexity compared to optimal detection approaches. Among the various IC schemes, successive hard decision IC (SIC) [1]-[3] and iterative hard decision IC (IHIC) [4] [5] utilize hard instead of soft decision values to further reduce the computational complexity. However, if a decoded bit sequence in a hard decision IC scheme contains errors, those errors can be propagated to other decoded bit sequences. In addition, if hybrid automatic repeat request (HARQ) [9]-[15] is employed for packet retransmission, the errors in the decoded bit sequences of previous transmissions can be transferred to the decoded bit sequences of future transmissions, owing to the HARQ combining process for retransmission. That is, the errors propagated to a packet by the hard decision IC schemes in previous transmissions become additional noise components of the packet in future transmissions, which disturbs the packet decoding success after retransmission. This phenomenon of error propagation throughout transmissions, which is termed "inter-transmission error propagation" for the first time in this paper, can degrade the error performances of MIMO systems employing HARQ with hard decision IC schemes. Furthermore, if multi-packet transmissions are allowed in an MIMO system employing HARQ [9] [10] [12]-[14], the inter-transmission error propagation of a packet can be transferred to other packets through the hard decision IC.

To prevent error propagation, error detection coding (EDC) schemes such as cyclic redundancy check (CRC) [16] [17] can be utilized for hard decision IC schemes [6] [7] [9]. Unlike the approach proposed in [8], in which EDC is utilized as a criterion to stop iteration, the hard decision IC schemes reported in [6], [7], and [9] perform IC when a decoded bit sequence is identified as error-free by the EDC. However, the hard decision IC schemes in [6] and [7] were designed for MIMO systems without HARQ, and can only be applied in the case of two simultaneously transmitted packets. Further, although the hard decision IC scheme in [9] was designed for MIMO systems employing HARQ, it is restricted to performing a CRC-based error detection procedure for every decoding iteration. Further, in [9], little effort was made to investigate the performance and operational characteristics of the utilized hard decision IC scheme.

Therefore, in this study, an EDC-aided IHIC (EDC-IHIC) scheme for general MIMO systems employing HARQ for multi-packet transmission [9] [10] [12]-[14] is developed and investigated. The EDC-IHIC scheme consists of detection, combining, decoding, error detection, and IC stages. The main difference between the proposed EDC-IHIC scheme and the existing SIC and IHIC schemes is i) the implementation of the error detection stage before the IC stage, which enables ii) IC operations based on error detection results for the EDC-IHIC scheme; this approach can allow iii) the EDC-IHIC scheme to eliminate the possibility of error propagation during IC operations. Specifically, the error detection stage of the EDC-IHIC scheme is executed after the decoding stage, which includes a number of decoding iterations, and the error detection stage determines whether the decoded bit sequence of each packet is error-free. Then, only packets having the decoded bit sequences identified as error-free are submitted to the IC stage for cancellation. Therefore, if the error detection result is perfect, 
EDC-IHIC completely eliminates the possibility of any kind of error propagation, including inter-transmission error propagation; this is in contrast to the existing SIC and IHIC schemes, which are not designed to consider error detection results during IC operations. This assumption becomes valid in practical systems employing HARQ that utilize a powerful EDC scheme to determine packet retransmission [15].

With regard to notation, throughout this paper, sets are represented in sans-serif, as in A, and vectors and matrices are denoted in boldface lowercase and uppercase, respectively. $\mathbf{I}_{A}$ denotes the $A \times A$ identity matrix. The superscripts $T, H$, and -1 denote the transpose, conjugate-and-transpose, and inverse operators, respectively. $\mathbb{E}[\cdot]$ denotes the mathematical expectation.

\section{Preliminaries}

\subsection{Definitions}

The main mathematical symbols used throughout this paper are as follows:

- $N_{\mathrm{p}}$ : the number of simultaneously transmitted packets in one transmission time slot;

- $N_{\mathrm{i}}\left(N_{\mathrm{o}}\right)$ : the number of transmitting (receiving) antennas;

- $P_{i}$ : the packet for transmitting the $i$ th data bit sequence;

- $T$ : the maximum permitted HARQ round for each packet;

- $p_{i, t}$ : the subpacket of $P_{i}$ for the $t$ th HARQ round;

- $\mathrm{P}_{k}$ : the set of indices of the packet transmitted during the $k$ th time slot. $\mathrm{P}_{k}(n)=i$ if $P_{i}$ is sent from the $n$th transmitting antenna during the $k$ th time slot;

- $t_{i, k}$ : the HARQ round of $P_{i}$ at the $k$ th time slot;

- $d_{\max }$ : the maximum number of turbo iterations;

- $f_{\max }$ : the maximum number of decoding iterations per turbo iteration;

- $c_{d}$ : the number of packets identified as non-error-free in the $d$ th turbo iteration;

- $e_{i, d}$ : the error detection result of $P_{i}$ in the $d$ th turbo iteration; $e_{i, d}=0(1)$ when $P_{i}$ is identified as error-free (non-error-free);

- $I_{i, t_{i, k}}$ : the set of log-likelihood ratios (LLRs) of all coded bits comprising $p_{i, t_{i, k}}$;

- $\mathrm{L}_{i, t_{i, k}}$ : the set of combined LLRs for all coded bits comprising $P_{i}$ to the $t_{i, k}$ th HARQ round;

- $\mathrm{B}_{i, t_{i, k}}$ : the set of the bit sequence decoded using $\mathrm{L}_{i, t_{i, k}}$.

\subsection{System model}

Consider an $N_{\mathrm{i}} \times N_{\mathrm{o}}\left(N_{\mathrm{i}} \leq N_{\mathrm{o}}\right)$ MIMO system employing HARQ for multi-packet 
transmission as illustrated in Fig. 1, where $N_{\mathrm{i}}=N_{\mathrm{p}}$ and the $N_{\mathrm{p}}$ packets are transmitted separately from the different transmitting antennas. It is assumed that channel state information (CSI) is fully available at the receiver, but not available at the transmitter. At the transmitter, the data bit sequence of each $P_{i}$ is encoded by EDC encoders employing, e.g., CRC codes, and by error correction coding (ECC) encoders employing, e.g., turbo codes. It is worth mentioning that the EDC encoder can be omitted from the transmitter if the utilized error correction code has strong error detection capability, e.g., in the case of low-density parity-check (LDPC) codes [14] [16]. Then, each $p_{i, t}$ for $1 \leq t \leq T$ is generated from the encoded and modulated bit sequence of $P_{i}$. Without loss of generality, each $p_{i, t}$ is assumed to contain one transmit symbol. Let $\mathbf{s}_{k}=\left[s_{k, 1} \cdots s_{k, N_{\mathrm{i}}}\right]^{T}$ with $\mathbb{E}\left[\mathbf{s}_{k} \mathbf{s}_{k}^{H}\right]=\mathbf{I}_{N_{\mathrm{i}}}$ be the $N_{\mathrm{i}} \times 1$ transmit signal vector at the $k$ th time slot, where each $s_{k, n}$ is the transmit symbol for the subpacket of $P_{\mathrm{P}_{k}(n)}$ at the $k$ th time slot. Then, the $N_{\mathrm{o}} \times 1$ receive signal vector $\mathbf{r}_{k}$ for $\mathbf{s}_{k}$ is written as

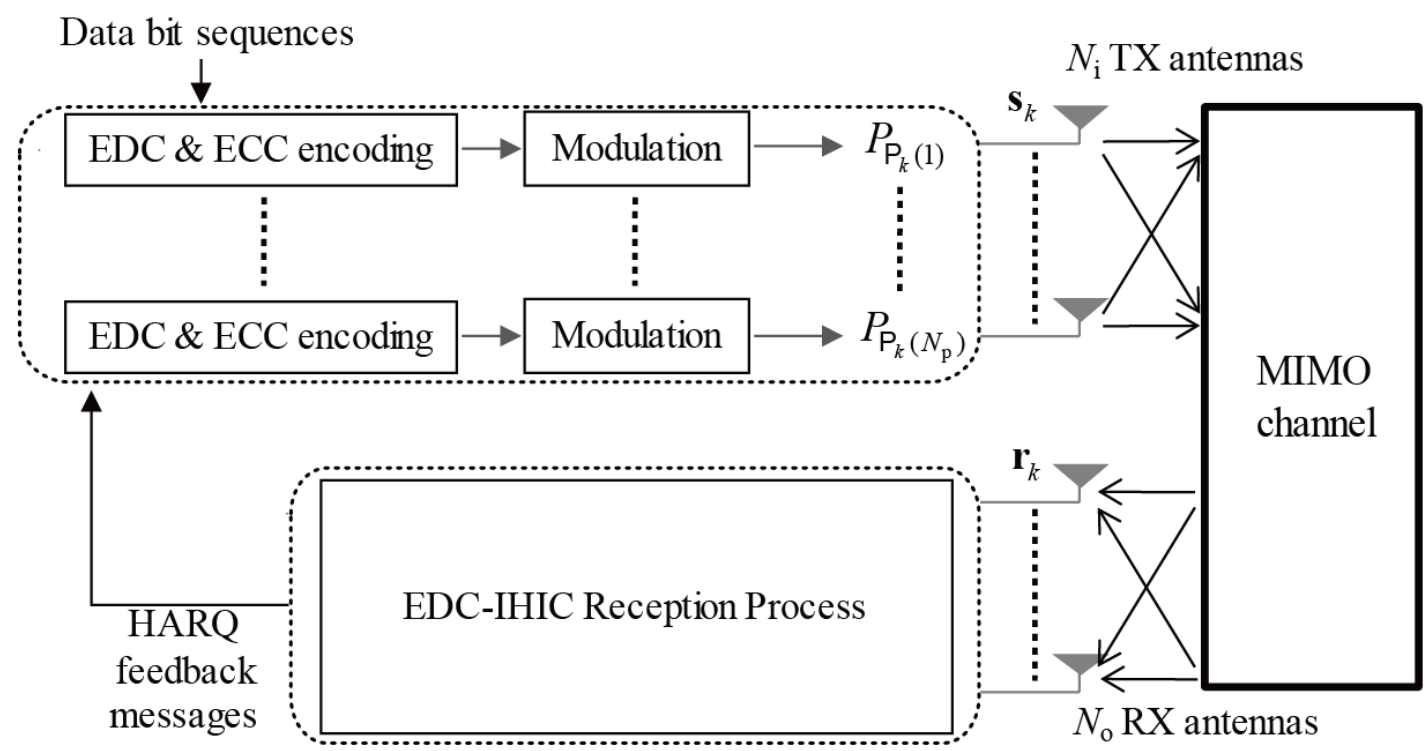

Fig. 1. System model block diagram.

$$
\mathbf{r}_{k}=\mathbf{H}_{k} \mathbf{s}_{k}+\mathbf{n}_{k},
$$

where $\mathbf{H}_{k}$ is the $N_{0} \times N_{\mathrm{i}}$ MIMO channel matrix and $\mathbf{n}_{k}$ is the $N_{\mathrm{o}} \times 1$ zero-mean additive white complex Gaussian noise vector with $\mathbb{E}\left[\mathbf{n}_{k} \mathbf{n}_{k}^{H}\right]=\sigma^{2} \mathbf{I}_{N_{\mathrm{o}}}$. Therefore, the average signal-to-noise ratio (SNR) is defined as $1 / \sigma^{2}$. 
If decoding of $P_{i} \in \mathrm{P}_{k}$ fails when $t_{i, k}<T, p_{i, t_{i, k+1}}$ with $t_{i, k+1}=t_{i, k}+1$ is transmitted during the $(k+1)$ th time slot. If $P_{i} \in \mathbf{P}_{k}$ is decoded successfully or $t_{i, k}=T$, the first subpacket of a new packet containing a new data bit sequence is transmitted during the $(k+1)$ th time slot.

\section{EDC-IHIC scheme}

In MIMO systems with multi-packet transmission, if an IC technique is employed at the receiver, the decoding result of each packet is related to those of the other packets. More specifically, if the decoding success probability of a packet is high (even neglecting the IC procedures), there is a high possibility that the interference from the packet with the other packets can be eliminated correctly, which will improve the decoding success probability of the other packets. On the other hand, if the decoding success probability of a packet is low (ignoring the IC procedures), there is a high possibility that the interference from the packet with the other packets will be eliminated incorrectly; this will degrade the decoding success probability of the other packets. Thus, a close relationship exists between the decoding results of the packets in MIMO systems for multi-packet transmission, when an IC technique is employed at the receiver.

Next, we assume that HARQ procedures are utilized for MIMO systems with multi-packet transmission employing an IC technique at the receiver. In general, the decoding success probability of a packet under HARQ procedures increases with the number of retransmissions. This increases occurs for many reasons, such as the increased diversity order, received SNR, and number of redundancy bits. However, if a packet experiences an incorrect IC operation related to some other packet(s), the former packet will suffer from the errors newly generated by the incorrect IC operation, as well as the existing interference originating from the other packet(s). In addition, the errors in the decoded bit sequences of previous transmissions can be transferred to the decoded bit sequences of future transmissions, because of the HARQ combining process for retransmission. That is, the occurrence of an incorrect IC operation at the early stage of the HARQ transmission process for a packet can degrade the error performance for that packet until the last stage of its HARQ transmission process. Furthermore, because of the nature of multi-packet transmission, such inter-transmission error propagation for a packet can be transferred to any other packets in any packet transmission time slot, once an incorrect IC operation has occurred. Therefore, it is important to prevent incorrect IC operation to obtain fine system performance, especially for MIMO systems employing HARQ for multi-packet transmission. 


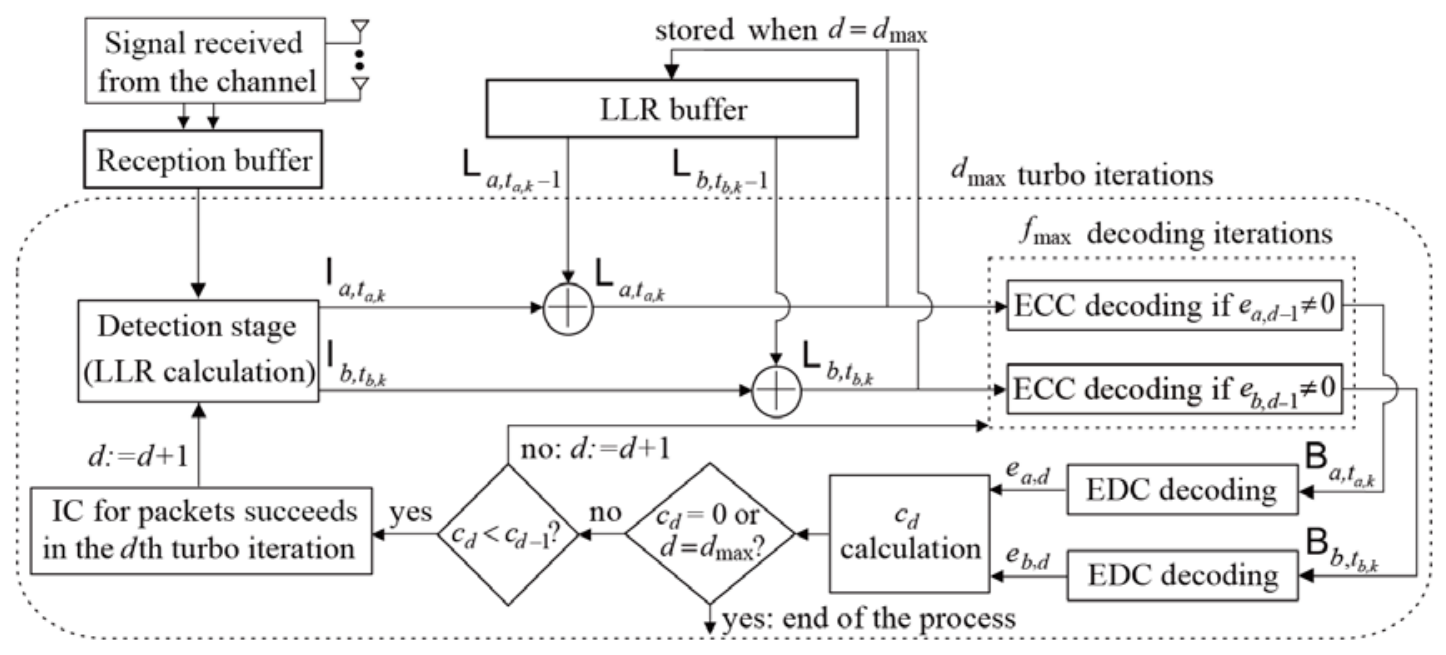

Fig. 2. EDC-IHIC receiver block diagram for $N_{\mathrm{p}}=2$.

To prevent incorrect IC operation, an EDC-IHIC scheme for MIMO systems employing HARQ for multi-packet transmission is developed in this study. The proposed EDC-IHIC scheme is designed to prevent incorrect IC operation by utilizing the error detection result for each packet in every turbo iteration. That is, if a packet is identified as non-error-free, that packet will not be submitted to the IC operation stage. Fig. 2 is the receiver block diagram of the EDC-IHIC scheme for MIMO systems employing HARQ for multi-packet transmission, assuming bit-level combining [10].

The reception procedures of the proposed EDC-IHIC scheme at the $k$ th packet transmission time slot are now explained in detail. The proposed EDC-IHIC scheme consists of detection, combining, decoding, error detection, and IC stages. Before the first turbo iteration, the initialization procedures for the iterative reception procedures are implemented. Let $\left\{\mathbf{r}_{k}, \mathbf{H}_{k}\right\}$ denote the receive signal vector and channel matrix at the $k$ th packet transmission time slot, respectively. Further, let $\left\{\mathbf{r}_{k, d}, \mathbf{H}_{k, d}\right\}$ be the receive signal vector and channel matrix utilized during the $d$ th turbo iteration at the $k$ th packet transmission time slot, respectively. As no IC operation can be performed before the first turbo iteration, $\mathbf{r}_{k, 1}=\mathbf{r}_{k}$ and $\mathbf{H}_{k, 1}=\mathbf{H}_{k}$. Further, the number of packets identified as non-error-free before the first turbo iteration, $c_{0}$ is set to $N_{\mathrm{p}}$ before the iterative procedures are initialized.

Then, the $d$ th turbo iteration of the proposed EDC-IHIC scheme begins with the detection stage. The detection stage is implmented for the non-error-free packets until the $(d-1)$ th turbo iteration, i.e., $p_{i, t_{i, k}}$ for all $i \in \mathrm{P}_{k}$ with $e_{i, d-1}=1$. First, the filtered output for the transmit symbols in the subpackets of the non-error-free packets, $\hat{\mathbf{x}}_{k, d}$ is obtained. For example, in the case of a linear minimum mean-square-error (LMMSE) filter, $\hat{\mathbf{x}}_{k, d}$ is obtained as 


$$
\hat{\mathbf{x}}_{k, d}=\mathbf{F}_{k, d} \mathbf{r}_{k, d}=\left(\mathbf{H}_{k, d}^{H} \mathbf{H}_{k, d}+\sigma^{2} \mathbf{I}_{c_{d}}\right)^{-1} \mathbf{H}_{k, d}^{H} \mathbf{r}_{k, d},
$$

where $\mathbf{F}_{k, d}$ is the LMMSE filter matrix. Then, the set of LLRs of all coded bits comprising subpacket $p_{i, t_{i, k}}$, i.e., $I_{i, t_{i, k}}$, can be calculated using $\hat{\mathbf{x}}_{k, d}$ and $\mathbf{F}_{k, d}$ as in [5]; this is the end of the detection stage.

Following generation of $\mathrm{I}_{i, t_{i, k}}$ in the detection stage, the combining stage is implemented. In the combining stage, LLR-level combining is implemented for the non-error-free packets until the $(d-1)$ th turbo iteration, i.e., for all $i \in \mathrm{P}_{k}$ with $e_{i, d-1}=1$. Specifically, $\mathrm{I}_{i, t}$ for $1 \leq t \leq t_{i, k}$ are combined to obtain the set of combined LLRs for all coded bits comprising $P_{i}$ to the $t_{i, k}$ th HARQ round, $\mathrm{L}_{i, t_{i, k}}$; this is the end of the combining stage.

Using the $\mathrm{L}_{i, t_{i, k}}$ generated in the combining stage, the decoding stage is implemented. For all $i \in \mathrm{P}_{k}$ with $e_{i, d-1}=1$, the ECC decoding process is performed using $\mathrm{L}_{i, t_{i, k}}$ with $f_{\max }$ decoding iterations. After $f_{\max }$ decoding iterations have been conducted, the decoded bit sequence $\mathrm{B}_{i, t_{i, k}}$ is generated for $P_{i}$; this is the end of the decoding stage.

Then, using the $B_{i, t_{i, k}}$ from the decoding stage, the error detection stage is performed for all $i \in \mathrm{P}_{k}$ with $e_{i, d-1}=1$, i.e., for all non-error-free packets, until the $(d-1)$ th turbo iteration. Using the employed EDC scheme, e.g., CRC or parity-check equations of LDPC codes, error detection is performed to check whether $\mathrm{B}_{i, t_{i, k}}$ is error-free. If no errors are detected in $\mathrm{B}_{i, t_{i, k}}$, $e_{i, d}$ is set to 0 . Otherwise, $e_{i, d}$ is set to 1 .

Finally, the IC stage is implemented, based on the error detection results of the non-error-free packets until the $(d-1)$ th turbo iteration. If $c_{d}=\sum_{i \in P_{k}} e_{i, d}$ is equal to 0 , all packets are identified as error-free. In this case, no further iterations are required and the reception process is ended. Likewise, if $d$ reaches the maximum number of turbo iterations $d_{\max }$, the reception process is ended. Otherwise, if the number of non-error-free packets after the $d$ th turbo iteration $c_{d}$ is not changed from the previous value, i.e., $c_{d}=c_{d-1}$, no IC operation is performed for the current $d$ th turbo iteration. This is because all the packets identified as non-error-free at the previous iteration are still identified as non-error-free after the current iteration, and IC operation using non-error-free packets yields error propagations, which should be prevented to achieve good system performance. In this case, as no IC operation is performed, the detection and combining stages for the next $(d-1)$ th turbo iteration can be omitted. Therefore, $\mathbf{r}_{k, d+1}$ and $\mathbf{H}_{k, d+1}$ are set to $\mathbf{r}_{k, d}$ and $\mathbf{H}_{k, d}$, respectively, and the decoding stage is repeated with $d:=d+1$. 
Otherwise, if $c_{d}$ is changed from the previous value, i.e., $c_{d}<c_{d-1}$, at least one packet has been newly identified as error-free in the current $d$ th turbo iteration. In this case, IC operation is performed using the packet(s) newly identified as error-free. Specifically, for all $i \in \mathrm{P}_{k}$ with $e_{i, d-1}=1$ and $e_{i, d}=0$ (packets newly identified as error-free in the current $d$ th turbo iteration), the IC operation is implemented according to

$$
\mathbf{r}_{k, d+1}=\mathbf{r}_{k, d+1}-\mathbf{h}_{k, n} \hat{s}_{k, n},
$$

where $n$ is the transmitting antenna for $p_{i, t_{i, k}}$, i.e., $\mathrm{P}_{k}(n)=i, \mathbf{h}_{k, n}$ is the $n$th column of $\mathbf{H}_{k}$, and $\hat{s}_{k, n}$ is the regenerated transmit symbol from $\mathrm{B}_{i, t_{i, k}}$ for $p_{i, t_{i, k}}$. After the IC operation, all $\mathbf{h}_{k, n}$ utilized in (3) (the columns corresponding to the packets newly identified as error-free in the $d$ th turbo iteration) are excluded from $\mathbf{H}_{k, d}$, and the reformulated $\mathbf{H}_{k, d}$ is stored as $\mathbf{H}_{k, d+1}$ for future turbo iterations. Subsequently, $d$ is set to $d+1$. The next turbo iteration then begins with the detection stage to obtain the updated LLRs for the remaining packets through IC operation, using the packets identified as error-free. This is the end of the IC stage and the $d$ th turbo iteration.

The above reception procedures of the EDC-IHIC scheme are summarized as follows:

0) [Initialization] Set $\mathbf{r}_{k, 1}:=\mathbf{r}_{k}$ and $\mathbf{H}_{k, 1}:=\mathbf{H}_{k}$. In addition, set $d:=1, c_{0}:=N_{\mathrm{p}}$.

1) [Detection stage] For all $i \in \mathrm{P}_{k}$ and $e_{i, d-1}=1$, calculate $\mathrm{I}_{i, t_{i, k}}$ using $\hat{\mathbf{x}}_{k, d}$ and the corresponding $\mathbf{F}_{k, d}$.

2) [Combining stage] For all $i \in \mathrm{P}_{k}$ and $e_{i, d-1}=1$, combine $\mathrm{I}_{i, t}$ for $1 \leq t \leq t_{i, k}$ to obtain $\mathrm{L}_{i, t_{i, k}}$.

3) [Decoding stage] For all $i \in \mathrm{P}_{k}$ and $e_{i, d-1}=1$, implement the ECC decoding process using $\mathrm{L}_{i, t_{i, k}}$ with $f_{\max }$ decoding iterations, so as to generate $\mathrm{B}_{i, t_{i, k}}$.

4) [Error detection stage] For all $i \in \mathrm{P}_{k}$ and $e_{i, d-1}=1$, perform error detection to check whether $\mathrm{B}_{i, t_{i, k}}$ is error-free. If no errors are detected in $\mathrm{B}_{i, t_{i, k}}$, set $e_{i, d}=0$. Otherwise, set $e_{i, d}=1$.

5) [IC stage] If $c_{d}:=\sum_{i \in P_{k}} e_{i, d}=0$ or $d$ reaches $d_{\max }$, stop the reception process. Otherwise, if $c_{d}=c_{d-1}$, set $\mathbf{r}_{k, d+1}:=\mathbf{r}_{k, d}$ and $\mathbf{H}_{k, d+1}:=\mathbf{H}_{k, d}$, and repeat from 3) with $d:=d+1$. If $c_{d}<c_{d-1}$, for all $i \in \mathrm{P}_{k}, e_{i, d-1}=1$, and $e_{i, d}=0$, implement IC as in (3). Then, set $\mathbf{H}_{k, d+1}:=\mathbf{H}_{k, d}$, exclude all $\mathbf{h}_{k, n}$ utilized in (3) from $\mathbf{H}_{k, d+1}$, and repeat from 1) with $d:=d+1$.

As shown in 5), a packet is utilized for IC only when it is newly identified as error-free during a turbo iteration. If no packets are newly identified as error-free during a turbo iteration, additional decoding iterations are performed without the IC, detection, and combining stages. Therefore, the probability of error propagation, including inter-transmission error propagation, in the EDC-IHIC scheme is determined by the undetected error probability of the EDC, which 
is completely eliminated if perfect error detection is assumed. The assumption of perfect error detection becomes valid for general systems employing HARQ that utilize a powerful EDC scheme to determine packet retransmission [15]. For example, the IEEE 802.16e standard uses CRC-CCITT with a size of a 16-bit size as the EDC for Chase combining [15]. The average undetected error probability of CRC-CCITT is less than $2^{-16}$ in the worst-case scenario, when the average bit error rate (BER) is $10^{-2}$ [16]. Therefore, if a powerful EDC is used for HARQ, the probability of error propagation in MIMO systems employing HARQ for multi-packet transmission becomes negligible under the EDC-IHIC scheme. In addition, if a linear block code such as LDPC is implemented as the ECC, the syndrome of a decoded bit sequence can be jointly utilized with the EDC for error detection [17], which can further decrease the undetected error probability.

As the detection stage in EDC-IHIC is executed only when the IC stage is executed in the previous turbo iteration, the computational complexity for the detection stages during $d_{\max }$ turbo iterations is dominated by the number of executed IC stages. The worst-case scenario in terms of computational complexity is the successful sequential decoding of one packet per turbo iteration, i.e., only one packet is newly identified as error-free in each turbo iteration. In that case, the size of the matrix utilized for the detection stage in the $d$ th turbo iteration $\mathbf{H}_{k, d}$ is $N_{\mathrm{o}} \times\left(N_{\mathrm{p}}-d+1\right)$, because $N_{\mathrm{p}}=N_{\mathrm{i}}$, and the computational complexity for the $d$ th turbo iteration is $\mathcal{O}\left(N_{\mathrm{o}}\left(N_{\mathrm{p}}-d+1\right)^{2}\right)$ [5] [6]. Therefore, the worst-case computational complexity for the detection procedures in (2) is $\mathcal{O}\left(N_{\mathrm{o}} \sum_{d=1}^{\min \left(d_{\max }, N_{\mathrm{p}}\right)}\left(N_{\mathrm{p}}-d+1\right)^{2}\right)$.

It is worth mentioning that error propagation prevention can also be applied to the existing SIC scheme, i.e., the EDC-aided SIC (EDC-SIC) scheme. In the EDC-SIC scheme, the error detection stage is incorporated between the decoding and IC stages, as in the EDC-IHIC scheme. Then, each packet is successively subjected to the decoding stage, and the decoded bit sequence of a given packet is only subjected to IC when it is identified as being error-free. Therefore, similar to the EDC-IHIC scheme, if the error detection result is perfect, the EDC-SIC scheme can also completely eliminate the possibility of any kind of error propagation, including inter-transmission error propagation. However, as a result of the nature of the successive decoding procedures, each packet in the EDC-SIC scheme experiences only one error detection stage before the IC operations. In contrast, each packet in the EDC-IHIC scheme can experience at most $d_{\max }$ error detection stages prior to the IC operations. That is, the EDC-IHIC scheme can provide more opportunities to cancel the packets identified as error-free than the EDC-SIC scheme. Therefore, it is expected that the error performance of the EDC-SIC scheme is inferior to that of the EDC-IHIC scheme, and further discussions on the EDC-SIC scheme are omitted from this paper.

\section{Numerical Results}

The LDPC code in [15] with a rate of 5/6 and size of 576 bits was considered as the mother code. CRC-24 was utilized as the EDC [17], and quadrature phase-shift keying (QPSK) modulation was considered. Chase combining [15] was implemented as the retransmission strategy, and a time-varying Rayleigh fading channel was considered. Antenna switching interleaving per transmit signal vector was utilized for transmissions, and the LMMSE filter 
was employed at the receiver. In addition to the hard decision IC schemes, the performance of the receiver having no IC operation (denoted as "NIC" below) was also evaluated as a control. We set $f_{\text {max }}=40$ for both IHIC and EDC-IHIC (in this section, the abbreviations are used to refer to the schemes themselves for improved brevity), and the number of decoding iterations for a packet was set to $d_{\max } \cdot f_{\max }$ for both NIC and SIC in order to maintain the maximum number of decoding iterations for the packets in each scheme.

Figs. 3 and $\mathbf{4}$ show comparisons of the average packet error rates (PERs) of NIC, SIC, IHIC, and EDC-IHIC for $N_{\mathrm{p}}=N_{\mathrm{i}}=N_{\mathrm{o}}=4$ and $d_{\max }=4$, where the average PER was defined as the ratio of the number of packets terminated in the last $T$ th HARQ round without decoding success to the total number of transmitted packets. For the results shown in Figs. $\mathbf{3}$ and $\mathbf{4}$, the normalized Doppler frequencies were set to $10^{-2}$ and $10^{-4}$, respectively. To obtain a reasonable average PER, numerical simulations were performed until 1000 packet errors were counted for each SNR point. Therefore, the $95 \%$ confidence interval values were $\pm 6.4 \%$ at maximum for the PER values at each SNR point [18].

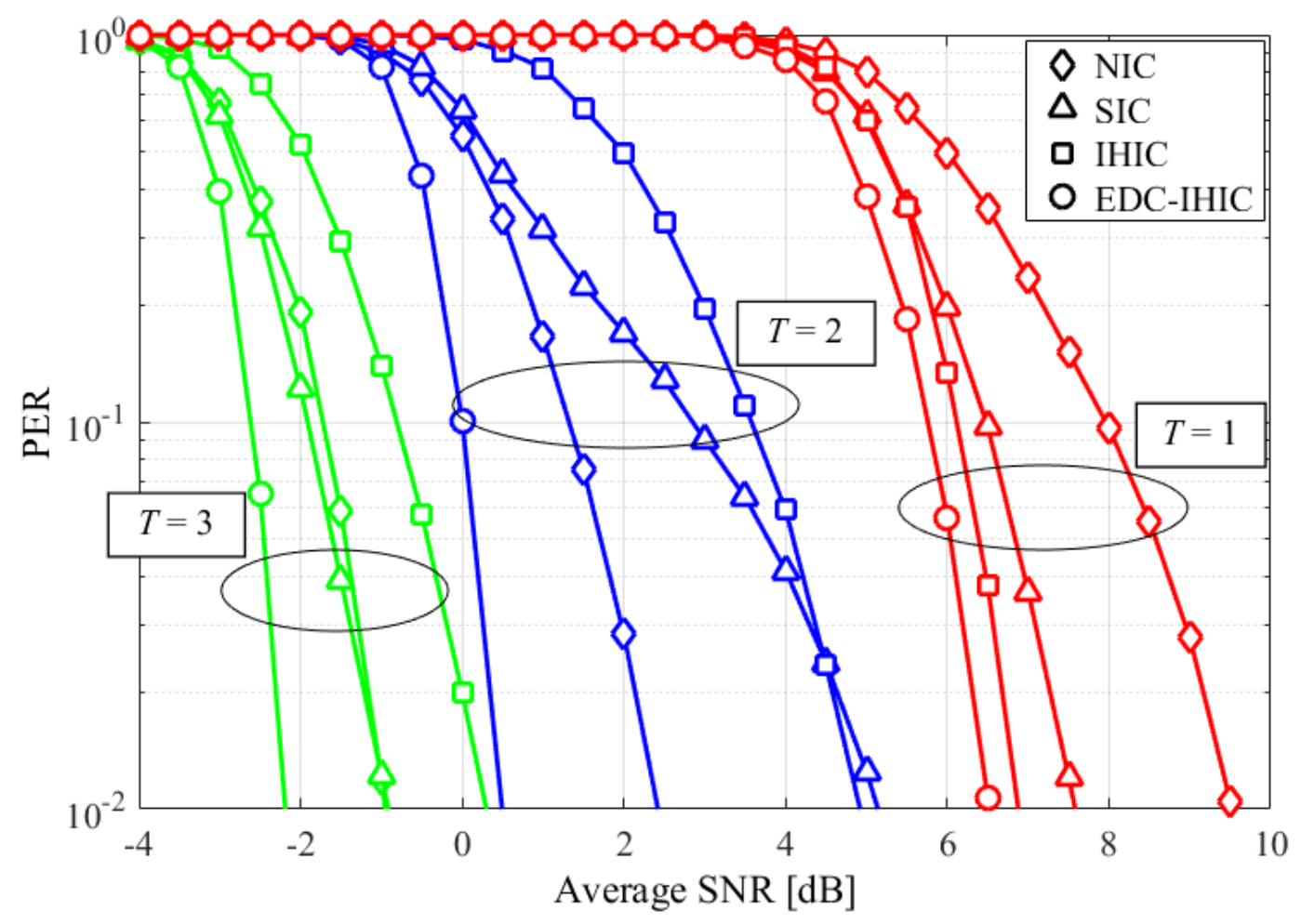

Fig. 3. Average PER comparisons for $N_{\mathrm{p}}=N_{\mathrm{i}}=N_{\mathrm{o}}=4, d_{\max }=4$, and normalized Doppler frequency of $10^{-2}$.

From Fig. 3, it is apparent that EDC-IHIC outperformed the other schemes in the fast fading channel regardless of $T$. Specifically, the SNR gains of EDC-IHIC over NIC, SIC, and IHIC at a PER of $10^{-2}$ were approximately $2.98,1.13$, and $0.42 \mathrm{~dB}$, respectively, for $T=1 ; 1.90$, 4.88, and $4.43 \mathrm{~dB}$, respectively, for $T=2$; and 1.30, 1.33, and $2.59 \mathrm{~dB}$, respectively, for 
$T=3$. SIC and IHIC exhibited superior PERs to NIC for the non-HARQ system with $T=1$. That is, in a non-HARQ system, the performance improvement due to correct IC operations can dominate the performance degradation due to incorrect IC operations. However, SIC and IHIC exhibited inferior PERs to NIC for the HARQ system with $T \geq 2$, especially in the low PER region. That is, in a HARQ system, existing hard decision IC schemes can suffer from performance degradation owing to inter-transmission error propagation, which cannot be overcome by an additional retransmission.

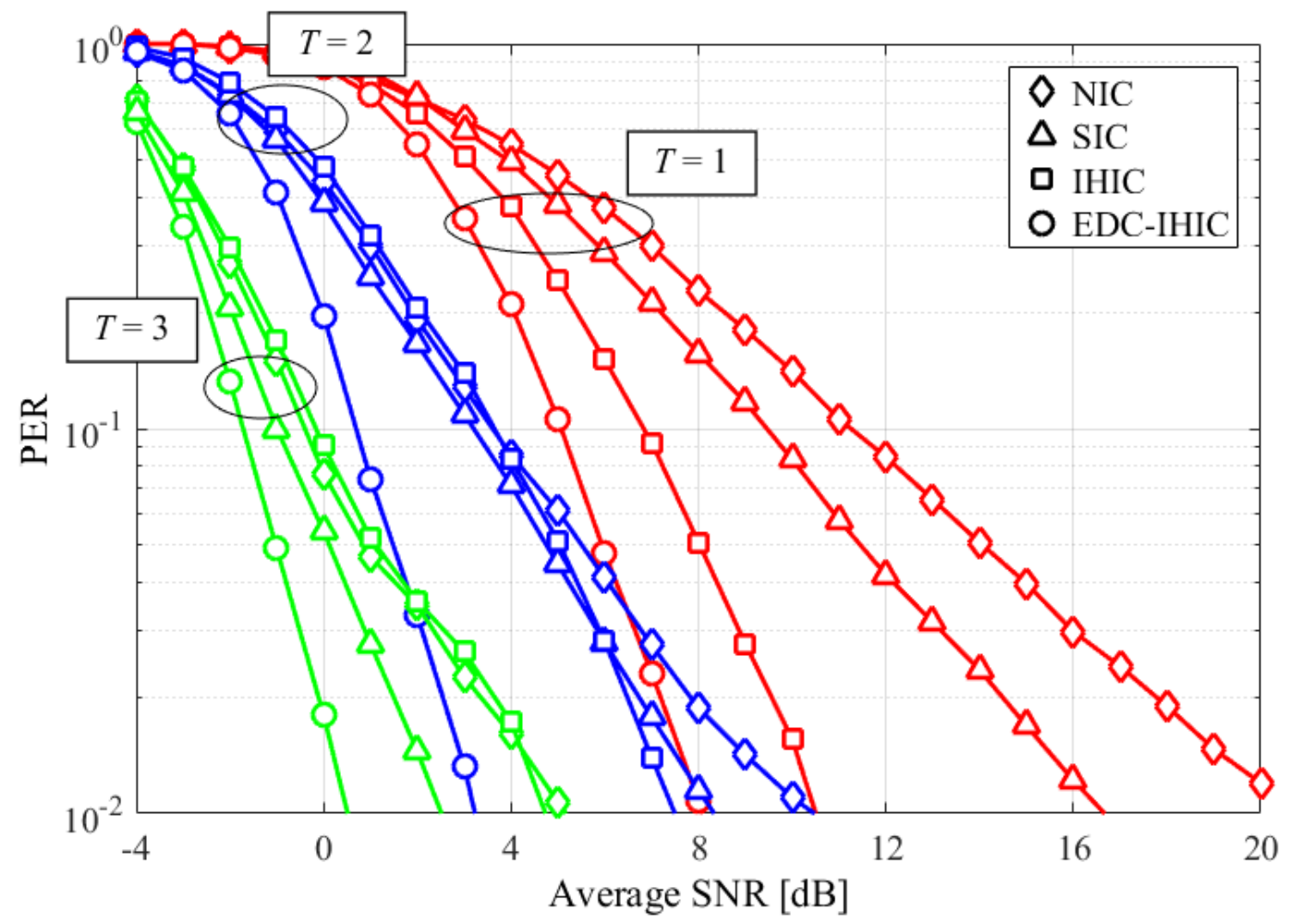

Fig. 4. Average PER comparisons for $N_{\mathrm{p}}=N_{\mathrm{i}}=N_{\mathrm{o}}=4, d_{\max }=4$, and normalized Doppler frequency of $10^{-4}$.

Similar to the results for the fast fading channel, Fig. 4 shows that EDC-IHIC outperformed the other schemes for the slow fading channel, regardless of $T$. Further, the SNR gains for the slow fading channel were, in general, further increased over those for the fast fading channel. This indicates that the technique of allowing only the correct IC operations (as in EDC-IHIC) can be a useful reception strategy to overcome the deep-fading impact. Specifically, the SNR gains of EDC-IHIC over NIC, SIC, and IHIC at a PER of $10^{-2}$ were approximately 12.7, 8.12, and $2.40 \mathrm{~dB}$, respectively, for $T=1 ; 7.26,5.03$, and $4.26 \mathrm{~dB}$, respectively, for $T=2$; and 4.66, 2.00 , and $4.22 \mathrm{~dB}$, respectively, for $T=3$. Unlike the fast fading channel, SIC and IHIC achieved similar or superior PERs to NIC for both non-HARQ and HARQ systems. However, because of the inter-transmission error propagation, the performance improvements of SIC and IHIC over NIC for the HARQ system with $T \geq 2$ were limited compared to those for the non-HARQ system with $T=1$. 


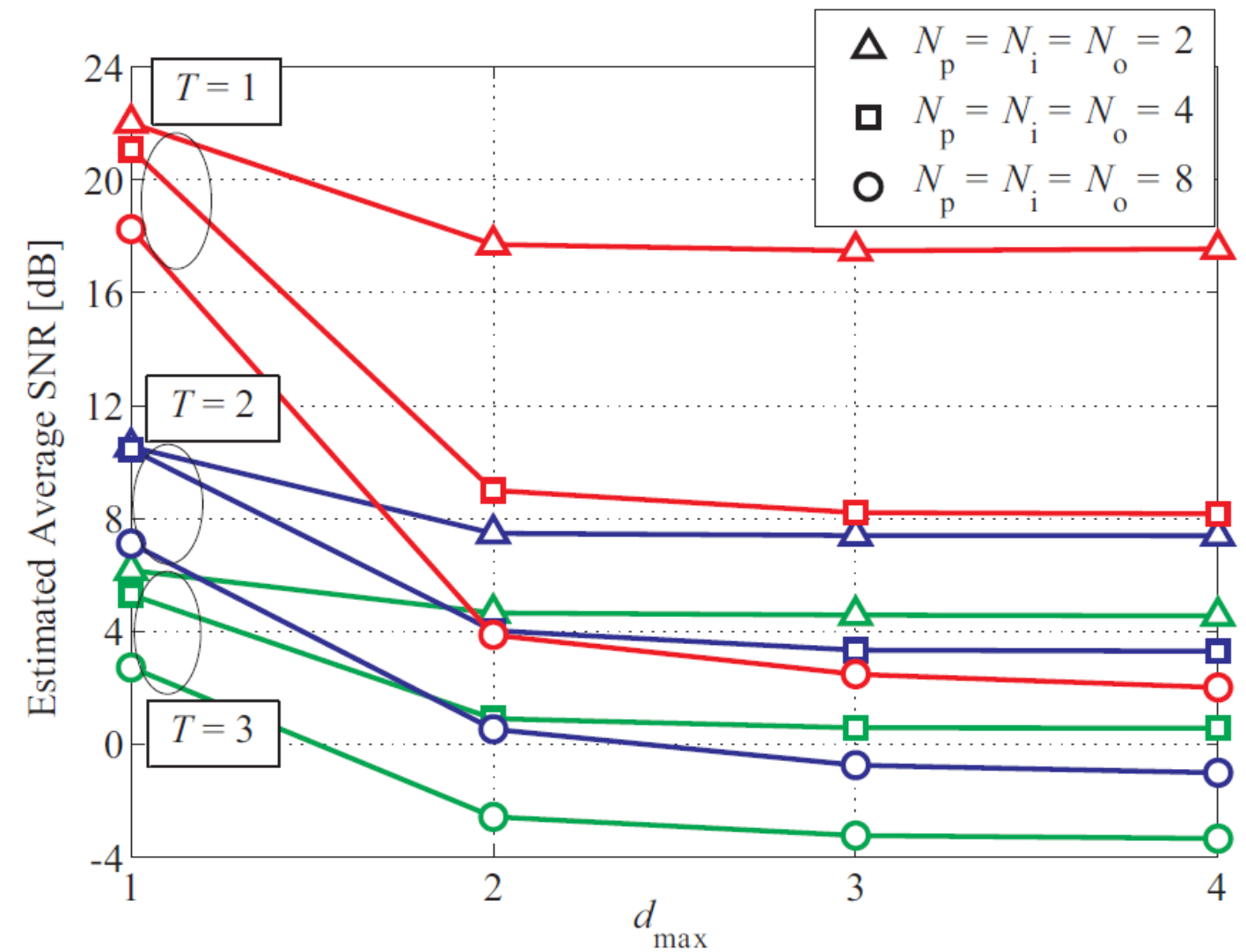

Fig. 5. Estimated average SNR values to achieve PER of $10^{-2}$ for EDC-IHIC with $N_{\mathrm{p}}=N_{\mathrm{i}}=N_{\mathrm{o}}$ and normalized Doppler frequency of $10^{-4}$.

Fig. 5 shows the estimated average SNR values that achieved a PER of $10^{-2}$ for EDC-IHIC, for $N_{\mathrm{p}}=N_{\mathrm{i}}=N_{\mathrm{o}}$ and a normalized Doppler frequency of $10^{-4}$. The case of $d_{\max }=1$ in Fig. 5 is equivalent to NIC having 40 decoding iterations. Compared with the results in Fig. 4, even EDC-IHIC with $d_{\max }=2$ was found to outperform SIC and IHIC with a larger $d_{\max }(=4)$ for $N_{\mathrm{p}}=4$. Further, for given $T$ and $d_{\max }$ values, the estimated average SNR values for EDC-IHIC continued to decrease as $N_{\mathrm{p}}$ increased. In other words, EDC-IHIC achieved superior PER with increasing $N_{\mathrm{p}}$ for given $T$ and $d_{\max }$ values. Specifically, the SNR gains of EDC-IHIC with $d_{\max }=4$ over EDC-IHIC with $d_{\max }=1$ were approximately $4.45,3.15$, and 1.61 dB for $T=1,2$, and 3, respectively, for $N_{\mathrm{p}}=2 ; 12.91,7.15$, and $4.72 \mathrm{~dB}$ for $T=1,2$, and 3, respectively, for $N_{\mathrm{p}}=4$; and 16.24, 8.12, and 6.06 dB for $T=1,2$, and 3, respectively, for $N_{\mathrm{p}}=8$. This is because the average number of packets decoded successfully before any IC operations in EDC-IHIC increased with $N_{\mathrm{p}}$ for a given average SNR. Therefore, in addition to the error propagation prevention achieved by EDC-IHIC, which includes inter-transmission error propagation, the performance improvements obtained under larger $N_{\mathrm{p}}$ render EDC-IHIC suitable for HARQ-employing MIMO systems with large input numbers, e.g., 
massive MIMO systems.

Table 1. Average numbers of complex multiplications of SIC, IHIC, and EDC-IHIC per transmit signal vector. Here, $N_{\mathrm{p}}=N_{\mathrm{i}}=N_{\mathrm{o}}=4$ and the normalized Doppler frequency is $10^{-4}$.

\begin{tabular}{|c|c|c|c|c|c|}
\hline$d_{\max }$ & 1 & 2 & 3 & 4 & 5 \\
\hline \hline$C_{\text {SIC }}$ & \multicolumn{5}{|c|}{} \\
\hline$C_{\text {IHIC }}$ & 288 & 384 & 672 & 576 & 672 \\
\hline$C_{\text {EDC-IHIC }}$ & 288 & 452.1 & 506.9 & 510.8 & 513.2 \\
\hline
\end{tabular}

Finally, in Table 1, the average numbers of complex multiplications of the hard decision IC schemes per transmit signal vector are listed for comparison, for the case of $N_{\mathrm{p}}=N_{\mathrm{i}}=N_{\mathrm{o}}=4$, a normalized Doppler frequency of $10^{-4}$, and $f_{\max }=40$. The operations in the hard decision IC schemes corresponding to (2) and (3) were evaluated. Here, $C_{\mathrm{SIC}}$, $C_{\text {IHIC }}$, and $C_{\text {EDC-IHIC }}$ represent the numbers of complex multiplications associated with SIC, IHIC, and the proposed EDC-IHIC, respectively. As regards EDC-IHIC, a greater number of IC and detection stages are performed, as the average number of packets decoded successfully before any IC operations increases; further, this number increases with the average SNR. Therefore, $C_{\text {EDC-IHIC }}$ was estimated for the case in which EDC-IHIC achieves a PER of $10^{-2}$ with $T=3$, which almost corresponds to the worst-case value with a sufficiently low PER. Note that, as the IHIC reception process does not rely on the decoding results, $C_{\text {IHIC }}$ is fixed regardless of PER and $T$. In addition, $C_{\mathrm{SIC}}$ is fixed regardless of PER, $T$, and $d_{\max }$, because of the successive procedures for all packets. Table 1 shows that $C_{\text {EDC-IHIC }}$ with $d_{\max }=5$ is comparable to $C_{\text {IHIC }}$ with $d_{\max }=3$ and, also, that $C_{\text {EDC-IHIC }}$ is always smaller than $C_{\mathrm{SIC}}$ in the simulation environments. The estimated $C_{\text {EDC-IHIC }}$ listed in Table 1 is close to its worst case value, and EDC-IHIC with $d_{\max }=2$ outperforms SIC and IHIC with larger $d_{\max }$, as in Fig. 5. Therefore, the proposed EDC-IHIC can be regarded as an effective hard decision IC scheme for HARQ employed MIMO systems with multi-packet transmission, in terms of computational complexity as well as error performance.

\section{Conclusion}

In this study, the EDC-IHIC scheme for MIMO systems employing HARQ for multi-packet transmission was developed and its use was investigated. The EDC-IHIC scheme eliminates the possibility of error propagation, including inter-transmission error propagation, and outperforms existing hard decision IC schemes while having comparable computational complexity. Because the use of a powerful EDC scheme is mandatory in systems employing HARQ to determine packet retransmission, the EDC-IHIC scheme can be applied to MIMO systems employing HARQ without the need for any additional redundancy. Furthermore, as no specific HARQ retransmission strategies are assumed in the system model, the EDC-IHIC scheme can be utilized for both Chase combining and incremental redundancy HARQ retransmission strategies. 
Although perfect CSI at the receiver was considered in this study for simplicity, in practical systems, both channel estimation procedures and signal detection procedures are required at the receiver. In such cases, the EDC-IHIC scheme can be utilized as the detection approach to facilitate joint channel estimation and detection procedures at the receiver, as in [19]. This topic could be investigated in future research.

\section{References}

[1] J. Choi, "Capacity/throughput optimization for H-BLAST with SC receiver over MIMO channels," IEEE Transactions on Wireless Communications, vol. 7, no. 3, pp. 1016-1024, March 2008. Article (CrossRef Link)

[2] J. Ketonen, M. Juntti, and J. R. Cavallaro, "Performance--complexity comparison of receivers for a LTE MIMO-OFDM system," IEEE Transactions on Signal Processing, vol. 58, no. 6, pp. 3360-3372, June 2010. Article (CrossRef Link)

[3] L. Wu, Y. Wang, J. Han, W. Chen, and L. Wang, "Optimal power allocation for wireless uplink transmissions using successive interference cancellation," KSII Transactions on Internet and Information Systems, vol. 10, no. 5, pp. 2081-2101, 2016. Article (CrossRef Link)

[4] A. Matache, C. Jones, and R. Wesel, "Reduced complexity MIMO detectors for LDPC coded systems," in Proc. of MILCOM 2004, pp. 1073--1079, October 2004. Article (CrossRef Link)

[5] D. N. Liu and M. P. Fitz, "Low complexity affine MMSE detector for iterative detection-decoding MIMO OFDM systems," IEEE Transactions on Communications, vol. 56, no. 1, pp. 150-158, January 2008. Article (CrossRef Link)

[6] Y. Li, J. H. Winters, and N. R. Sollenberger, "MIMO-OFDM for wireless communications: signal detection with enhanced channel estimation," IEEE Transactions on Communications, vol. 50, no. 9, pp. 1471-1477, September 2002. Article (CrossRef Link)

[7] G. Berardinelli, C. N. Manchón, L. Deneire, T. B. Sørensen, P. Mogensen, and K. Pajukoski, "Turbo receivers for single user MIMO LTE-A uplink," in Proc. of VTC 2009-SPRING, pp. 1-5, April 2009. Article (CrossRef Link)

[8] X. Mao, P. Amini, and B. Farhang-Boroujeny, "Markov chain Monte Carlo MIMO detection methods for high signal-to-noise ratio regimes," in Proc. of IEEE GLOBECOM 2007, pp. 3979-3983, November 2007. Article (CrossRef Link)

[9] Y. Whang, S. Park, H. Liu, "Effective interference level-based packet transmission for multiple-input multiple-output systems with hybrid automatic repeat request," IET Communications, vol. 9, no. 18, pp. 2208-2215, December 2015. Article (CrossRef Link)

[10] C. Bai, W. A. Krzymień, and I. J. Fair, "Hybrid-ARQ for layered space time MIMO systems with channel state information only at the receiver," IET Communications, vol. 4, no. 14, pp. 1765-1773, September 2010. Article (CrossRef Link)

[11] S. Park, Y. Whang, and S. Choi, "Extended detection for MIMO Systems with partial incremental redundancy based hybrid ARQ," IEEE Transactions on Wireless Communications, vol. 11, no. 10, pp. 3714-3722, October 2012. Article (CrossRef Link)

[12] S. Park, "Multiple retransmission strategy-based HARQ for MIMO systems with acknowledgment bundling," Electronics Letters., vol. 50, no. 8, pp. 637-639, April 2014. Article (CrossRef Link)

[13] S. Park and S. Choi, "Performance of symbol-level combining and bit-level combining in MIMO multiple ARQ systems," IEEE Transactions on Communications, vol. 64, no. 4, pp. 1517-1528, April 2016. Article (CrossRef Link)

[14] S. Park and S. Choi, "Multi-stage turbo equalization for MIMO systems with hybrid ARQ," Journal of Communications and Networks, vol. 18, no. 3, pp. 333-339, June 2016. Article (CrossRef Link)

[15] IEEE P802.16e, IEEE Standard for Local and Metropolitan Area Networks Part 16: Air Interface for Fixed and Mobile Broadband Wireless Access Systems, 2006 Article (CrossRef Link) 
[16] T. Fujiwara, T. Kasami, A. Kitai, and S. Lin, "On the undetected error probability for shortened Hamming codes,” IEEE Transactions on Communications, vol. 33, no. 6, pp. 570-574, June 1985. Article (CrossRef Link)

[17] T. K. Moon, Error Correction Coding: Mathematical Methods and Algorithms, John Wiley \& Sons, 2005. Article (CrossRef Link)

[18] J. Hamkins, "Confidence intervals for error rates observed in coded communications systems," IPN Progress Report, May 2015. Article (CrossRef Link)

[19] P. S. Rossi and R. Müller, "Joint twofold-iterative channel estimation and multiuser detection for MIMO-OFDM systems," IEEE Transactions on Wireless Communications, vol. 7, no. 1, pp. 4719-4729, January 2008. Article (CrossRef Link)

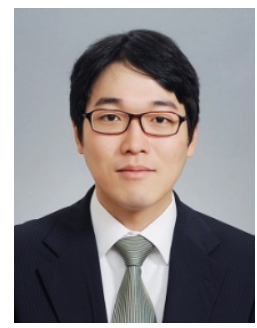

Sangjoon Park was born in Seoul, Korea, in 1981. He received the B.S., M.S., and Ph.D. degrees in electrical and electronic engineering from Yonsei University, Seoul, Korea, in 2004, 2006, and 2011, respectively. From August 2011 to February 2014, he was with LG Electronics as a Senior Research Engineer, participating in research on telematics and vehicular communication systems. From April 2014 to August 2016, he was a Research Professor with the School of Electrical and Electronic Engineering, Yonsei University. Since September 2016, he has been an Assistant Professor with the Department of Information and Communication Engineering, Wonkwang University. His main research interests include communication signal processing techniques, such as detection and equalization algorithms, hybrid ARQ and MIMO applications for wireless communications, error correcting codes and cryptography implementations, and other signal processing techniques. 